\title{
On the mutual relatedness of evenness measures
}

\author{
C. Ricotta ${ }^{1,3}$, E. De Zuliani ${ }^{1}$, A. Pacini ${ }^{2}$ and G. C. Avena ${ }^{1}$ \\ ${ }^{1}$ Department of Plant Biology, University of Rome "La Sapienza", Piazzale Aldo Moro 5, 00185 Rome, Italy. \\ 2 Department of Biology, University Roma Tre, Viale Marconi 446, 00146 Rome, Italy. \\ 3 Corresponding author: Tel.: +39-06-4991-2408; Fax:+39-06-4457-540; E-mail: carlo.ricotta@uniroma1.it
}

Keywords: Correlation, Evenness, Lorenz ordering, Principal component analysis.

\begin{abstract}
Several evenness measures have been proposed for quantifying the distribution of abundance among community species, but none seems to be generally preferred. Since these measures have the common objective of summarizing community structure, they may be expected to be intercorrelated. In this paper, seven standard measures of evenness were calculated for 65 sample plots of ruderal vegetation within the archaeological sites of Paestum and Venosa (southern Italy). Principal component analysis was used to identify the primary aspects of community structure being characterized by these seven indices. The first two principal components explained $96 \%$ of total variance. A comparison of the first two principal components with the analyzed measures of evenness provides insight into what aspects of community structure they are expressing. While the first principal component is most sensitive to the relative abundances of rare species, the second principal component is clearly associated to changes in the abundance of the dominant community species.
\end{abstract}

Abbreviation: PCA - Principal Component Analysis.

\section{Introduction}

The degree of equality among species relative abundances, called evenness, is a basic property of a biological community. Measures of evenness summarize the distribution of abundance among community species without regard to species names or labels. Maximum evenness (1.0) occurs for an equiprobable (i.e., uniform) species distribution, and the more relative abundances differ among the species the lower the evenness is. The concept of evenness is closely related to that of species diversity. It is generally agreed that diversity measures should combine two components: species richness (the number of species in the community, $N$ ) and evenness. High species richness and high evenness jointly imply high diversity.

Several evenness indices have been proposed (Taillie 1979, Smith and Wilson 1996). However, none seems to be generally preferred. Summarizing a large data set into a few numbers generally results in some loss of information. Therefore, some would argue that evenness indices conceal more than they reveal (Rousseau et al. 1999). However, ecological data are often multivariate of high dimension so there is a need for summarization. Since different evenness measures are attempting to quantify the same aspect of community structure, they can be expected to have a high degree of intercorrelation. In this paper, we analyze the mutual relatedness of seven standard evenness measures. Ideally, there is a small set of measures that characterize different aspects of the distribution of abundance among community species without being mutually redundant (Riitters et al. 1995, Basak et al. 2000). Therefore, the purpose of this study was to determine (i) the number of independent aspects of community structure that are summarized by these seven evenness measures, and (ii) the indices which best quantify these independent aspects of community structure.

\section{Requirements for an ecologically meaningful evenness measure}

Many authors (Taillie 1979, Routledge 1983, Smith and Wilson 1996) have proposed criteria that an index should satisfy in order to qualify as a measure of evenness. An ecologically acceptable measure of evenness should be reasonably simple to compute and applicable to any community independently of the underlying speciesabundance distribution (Alatalo 1981, Lande 1996). Furthermore, it should be well-defined mathematically in a way to be really useful in ecological applications. The foremost requirement for a meaningful evenness index is that it must be independent of the number of species 
(Smith and Wilson 1996). This requirement is based on the assumption that community diversity can be partitioned into two components, species richness and evenness. If the separation is incomplete, so that evenness is affected by the number of species, then differences in evenness values could result from differences in the species count rather than any fundamental difference in community organization (Sheldon 1969).

As a precise formulation for this notion of independence of species richness, Hill (1973) proposed that replication should not change the value of community evenness. Consider an $\mathrm{N}$-species community characterized by the relative abundance vector $\mathbf{p}=\left(p_{1}, p_{2}, \ldots, p_{N}\right)$ such that $0 \leq p_{i} \leq 1$ and $\Sigma p_{i}=1$. It seems reasonable that replicating the $N$-species sequence $n$-times (and renormalizing) should multiply richness by $n$ but leave evenness unchanged. Notice that this replication property is part of Taillie's (1979) more general requirement that an evenness index maintains the natural ordering introduced by the Lorenz curves used by economists to compare wealth distributions.

The Lorenz curve is obtained by plotting the cumulative species relative abundances as abscissa against corresponding cumulative proportions of species as ordinates. Arrange the components of the species relative abundance vector $\mathbf{p}$ of a given community in descending order so that the ranked abundance vector $\mathbf{p} \#=$ $\left(p_{1}^{\#}, p_{2}^{\#}, \ldots, p_{N}^{\#}\right)$ is obtained, where $p_{1}^{\#} \leq p_{2}^{\#} \leq \ldots \leq p_{N}^{\#}$. The Lorenz curve is then defined as the polygonal path joining the successive points: $\pi_{0}=(0,0), \pi_{1}=\left(p_{1}{ }^{\#}, 1 / N\right)$, $\pi_{2}=\left(p_{1}{ }^{\#}+p_{2}{ }^{\#}, 2 / N\right), \ldots, \pi_{\mathrm{N}}=\left(p_{1}{ }^{\#}+p_{2}{ }^{\#}+\ldots+p_{N}^{\#}, N / N\right) \equiv$ $(1,1)$ (Figure 1). The resulting diagram is similar to the intrinsic diversity profile proposed by Patil and Taillie $(1979,1982)$ for defining the concept of intrinsic diversity order: both use as abscissa the cumulative species relative abundances. However, the intrinsic diversity profile uses as ordinate the cumulative number of species, whereas the Lorenz curve uses as ordinate the cumulative proportion of species. Patil and Taillie $(1979,1982)$ defined community A to be intrinsically more diverse than community $\mathrm{B}$ without reference to indices, provided B leads to A by a finite sequence of forward transfers of abundance (for mathematical details, see Patil and Taillie 1979, 1982). Following this definition, the hypothetical community A is intrinsically more diverse than community B if and only if community A has its intrinsic diversity profile everywhere above that of community B. Notice that the ordering is only partial in that two communities need not be intrinsically comparable. In this latter case, the intrinsic diversity profiles of both communities cross one another. Similarly, community A is intrinsically more even than community B if and only if community A has its Lorenz curve everywhere above that of community B. Consequently, a measure of evenness $E$ that is invariant under species replication maintains the Lorenz ordering provided that $E$ is consistent with the intrinsic diversity ordering when restricted to communities with the same number of species (Taillie 1979). For instance, when diversity comparisons are restricted to communities with the same number of species, since there is no fundamental difference between diversity and evenness when species richness is held constant, the intrinsic diversity ordering is identical to the corresponding Lorenz ordering.

\section{Index selection}

We consider seven standard evenness indices that are consistent with the Lorenz ordering. These indices include:
Figure 1. Lorenz curve for an artificial five-species community with relative abundances $0.40,0.25,0.20$, $0.10,0.05$. Dotted line represents the Lorenz curve for a perfect even community, i.e., for a community where $p_{i}=p_{j}$ for all species pairs $i, j=1,2, \ldots, N$.

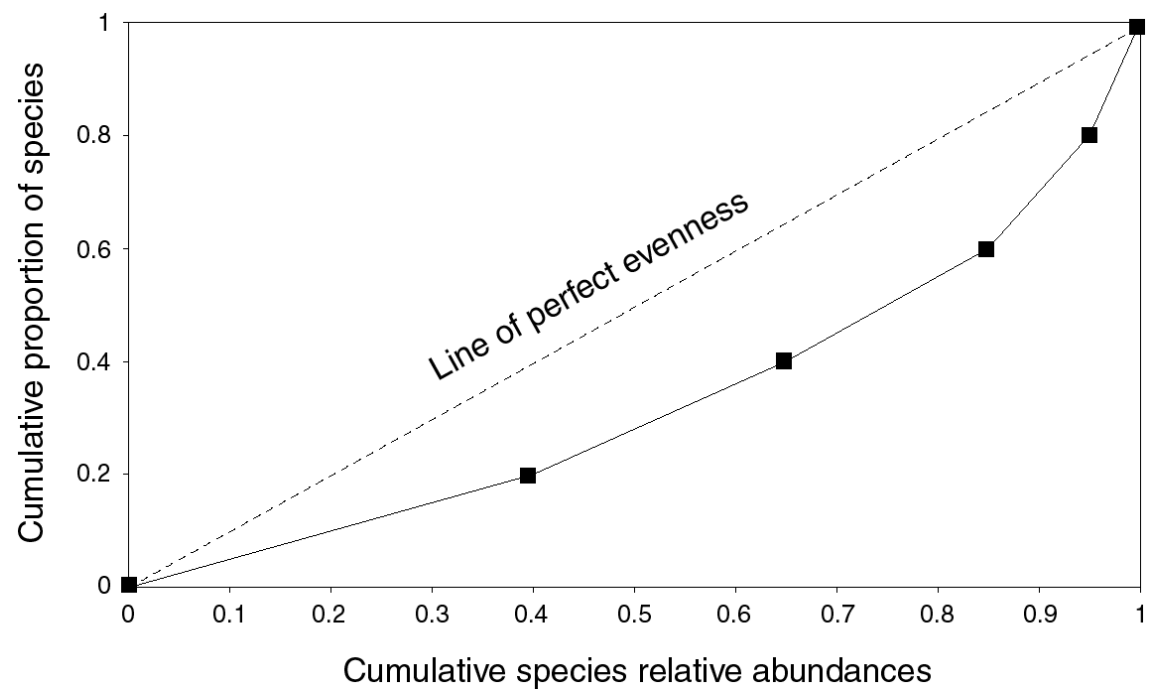


The index of Bulla (1994):

$O=\sum_{i=1}^{N} \min \left(p_{i}, 1 / N\right)$

The Gini index, i.e., twice the area under the Lorenz curve (Taillie 1979):

$$
I=\left(2 \sum_{i=1}^{N} i^{\#} p_{i}^{\#}-1\right) / N
$$

where $i^{\#}$ denotes the rank of the $i$-th species within the ranked abundance vector $\mathbf{p}^{\#}$. Notice that $I$ is related to Camargo's (1992) dominance index $d^{\prime}$ by the simple relation $I=1-d^{\prime}$, where

$d^{\prime}=\sum_{i=1}^{N} \sum_{j \geq i}^{N}\left|p_{i}^{\#}-p_{j}^{\#}\right| / N$

and $p_{i}^{\#}$ and $p_{j}^{\#}$ are the ranked relative abundances of the $i$-th and $j$-th species, respectively so that, $i^{\#}>j^{\#}$.

Two indices proposed by Taillie (1979):

$G M / A M$

and

$H M / A M$

where $A M, G M$ and $H M$ are the algebraic mean, the geometric mean and the harmonic mean of the species abundances, respectively.

Three moments of Hill's (1973) parametric evenness family

$E_{\alpha, 0}=N^{-1}\left(\sum_{i=1}^{N} p_{i}^{\alpha}\right)^{\frac{1}{1-\alpha}}$

where $\alpha$ is a parameter that ranges between 0 and $\infty$ (Taillie 1979, Ricotta and Avena 2000):

$E_{1,0}=(\exp H) N^{-1}$

where $H$ is Shannon's entropy

$E_{2,0}=D^{-1} N^{-1}$

where $D$ is Simpson's dominance index , and

$\mathrm{E}_{\infty}, 0=p_{\max }{ }^{-1} N^{-1}$

where $p_{\max }$ is the proportional abundance of the most frequent species.

\section{Methods and results}

In spring 1998 and 1999, 65 square sample plots $2 \mathrm{~m}$ $\mathrm{x} 2 \mathrm{~m}$ in size were randomly selected to sample the vegetation that colonize the archaeological sites of Paestum and Venosa (southern Italy). Each plot was subdivided into 10 by 10 subunits. For each plot, presence/absence of all vascular plants in each of the 100 subunits was recorded. The number of species per sample plot varied from 8 to 22. The complete data are available from the authors upon request. Vegetation cover values within each sample plot was first normalized to sum to unity. Next, the selected evenness measures $O, I, G M / A M$, $H M / A M, E_{1,0}, E_{2,0}$ and $E_{\infty}, 0$ were computed for each of the 65 sample plots.

Simple summary statistics for the selected evenness measures are shown in Table 1. The correlation coefficients of all pairs among the 7 measures are displayed in the triangular matrix of Table 2 .

Peet (1974) and Magurran (1988) distinguished two groups of diversity and evenness indices. Type I indices are most sensitive to the relative abundances of rare species, while Type II indices are most affected by changes in the abundance of the dominant species. Within this framework, in Table 2, all genuine Type I measures (e.g., $O, I, G M / A M$ and $E_{1,0}$ ) form a group where all withingroup coefficient of correlations are larger than 0.9. Conversely, the remaining measures $H M / A M, E_{2,0}$ and $E_{\infty}, 0$ reflect dominance to varying degrees and show lower pairwise coefficients of correlation. For instance, while $E_{\infty}, 0$ is clearly related to the abundance of the commonest community species, $H M / A M$ and $E_{2,0}$ may be considered as 'hybrid' indices with intermediate characteristics between Type I and Type II measures.

The intrinsic dimensionality of the selected evenness measures was further assessed using standardized principal component analysis (PCA) available through the SYN-TAX 5.02 package (Podani 1993).

The component scores of each evenness measure (i.e., correlations with axes) for the first two principal components are represented in Figure 2. These two com-

Table 1. Summary statistics for the selected evenness measures. Std. Dev. = standard deviation; $\mathrm{CV}=$ coefficient of variation.

$\begin{array}{llcc} & \text { Mean } & \text { Std. Dev. } & \text { CV } \\ \text { O } & 0.537 & 0.089 & 0.167 \\ \mathrm{I} & 0.425 & 0.091 & 0.215 \\ \mathrm{GM} / \mathrm{AM} & 0.507 & 0.117 & 0.231 \\ \mathrm{HM} / \mathrm{AM} & 0.311 & 0.104 & 0.335 \\ \mathrm{E}_{1,0} & 0.529 & 0.114 & 0.215 \\ \mathrm{E}_{2,0} & 0.368 & 0.107 & 0.290 \\ \mathrm{E}_{\infty, 0} & 0.205 & 0.064 & 0.311\end{array}$


Table 2. Pairwise correlation coefficients among the selected evenness measures $(n=65)$.

$\begin{array}{lccccccc} & 0 & I & \mathrm{GM} / \mathrm{AM} & \mathrm{HM} / \mathrm{AM} & \mathrm{E}_{1,0} & E_{2,0} & E_{\infty, 0} \\ \mathrm{O} & 1.000 & 0.965 & 0.962 & 0.837 & 0.954 & 0.844 & 0.557 \\ \mathrm{I} & & 1.000 & 0.979 & 0.889 & 0.971 & 0.885 & 0.657 \\ \mathrm{GM} / \mathrm{AM} & & & 1.000 & 0.937 & 0.953 & 0.834 & 0.567 \\ \mathrm{HM} / \mathrm{AM} & & & & 1.000 & 0.818 & 0.671 & 0.419 \\ \mathrm{E}_{1,0} & & & & & 1.000 & 0.954 & 0.725 \\ \mathrm{E}_{2,0} & & & & & & 1.000 & 0.862 \\ \mathrm{E}_{\infty, 0} & & & & & & & 1.000\end{array}$

Figure 2. Principal components ordination of evenness measures on the first two axes.

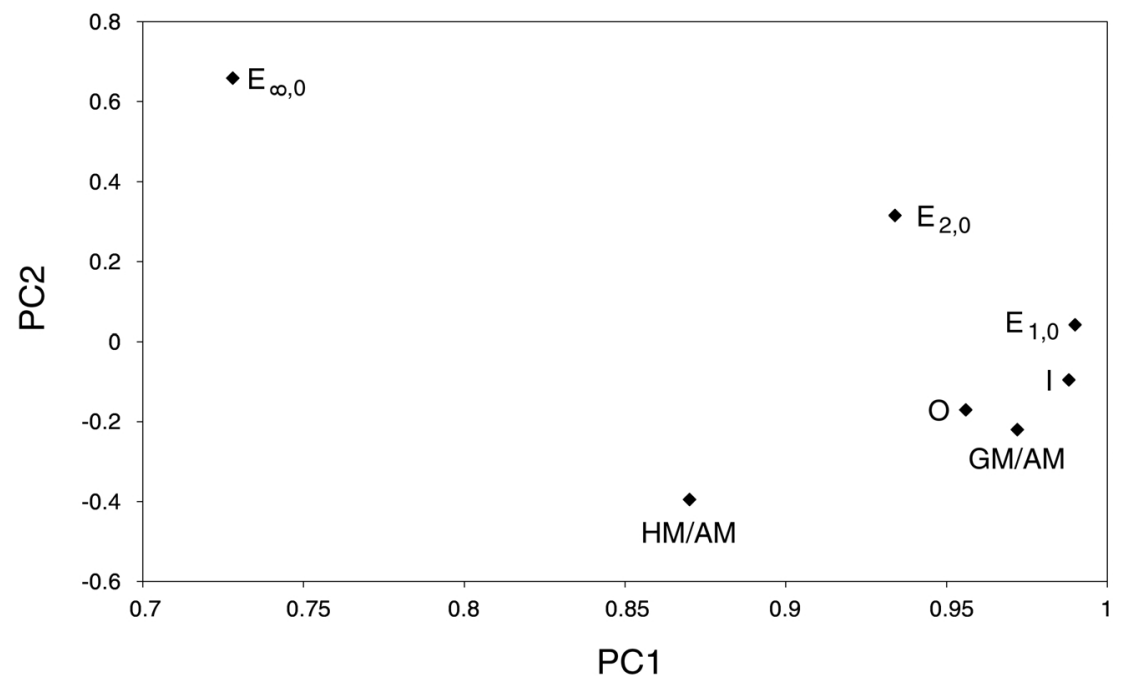

Due to the high redundancy among the indices analyzed, the scores of each measure of evenness on the first principal component are generally very high. As shown in Figure 2, $E_{1,0}$ is the measure with the highest loading on the first principal component, followed by the Gini index $I$. As mentioned above, the second principal component is strongly associated only with the $E_{\infty}, 0$ index. Therefore, it seems reasonable to interpret the second principal component as representing changes in the abundance of the dominant community species.

However, there are also different criteria for selecting the most suitable measure to be used as a surrogate of the first principal component. For example, as suggested by Magurran (1988), an effective evenness measure must be able to distinguish between communities with similar species abundance structures. The index effectiveness will therefore depend on the range of values it takes over the communities of interest. In this view, as shown in Table $1, H M / A M$ is the measure with the highest coefficient of variation $(\mathrm{CV}=0.335)$.

One additional criterion proposed by He and Orlóci (1993) is that the selected evenness measure be interpretable in information-theoretical terms. In this view, the evenness measures derived from Hill's (1973) generalized evenness family $E_{\alpha}, 0$ are monotone transformation of Rényi's (1970) measure of divergence 




Figure 3. Evenness values for all possible abundance relationships in two-species communities for an ideal evenness measure sensu Molinari (1989).

$H_{\alpha}(\mathbf{q} \| \mathbf{p})=\frac{1}{\alpha-1} \log \sum_{i=1}^{N} \frac{q_{i}^{\alpha}}{p_{i}^{\alpha-1}}$

where $\mathbf{p}=\left(p_{1}, p_{2}, \ldots, p_{N}\right)$ denotes an a priori (i.e., reference) relative abundance vector, and $\mathbf{q}=\left(q_{1}, q_{2}, \ldots, q_{N}\right)$ the observed abundance vector. $H_{\alpha}(\mathbf{q}|| \mathbf{p})$ measures the information gain on $\mathbf{p}$ contained in the observation of $\mathbf{q}$ and is defined only for $p_{i}>0(i=1,2, \ldots, N)$ and if there is a one-to-one correspondence between the elements of $\mathbf{p}$ and $\mathbf{q}$ (Rényi 1970). For a perfectly even reference vector $\mathbf{p}$ (i.e., if $p_{i}=p_{j}$ for all species pairs $i, j=1,2, \ldots, N$ ), Hill's (1973) parametric evenness $E_{\alpha, 0}$ is related to $H_{\alpha}(\mathbf{q} \| \mathbf{p})$ by the simple expression (Ricotta and Avena, unpublished data):

$H_{\alpha}(\mathbf{q} \| \mathbf{p})=-\log E_{\alpha, 0}$

Therefore, both measures $E_{1,0}$ and $E_{2,0}$ might be adequate surrogates of the first principal component within the context of a general theoretical framework based on information theory.

Finally, Molinari (1989) suggested that, in cases of two-species assemblages, an ecologically acceptable evenness index should keep a linear relationship between minimum and maximum evenness. Allow that a given evenness measure $E$ ranges between zero when the evenness is minimum (i.e., if there is a species with proportional abundance approaching 1 , then the abundances of all other species approach zero) and unity when evenness is maximum. For a community composed of two species, we would assign an evenness value close to zero to the case where $p_{i} \rightarrow 0$ and $p_{j} \rightarrow 1$. Conversely, we would assign an evenness value of 1.0 to the case where $p_{i}=p_{j}=$ 0.5 . For an ideal evenness measure sensu Molinari (1989), because the $p_{i}=0.25 ; p_{j}=0.75$ case is the intermediate between the extreme cases $p_{i} \rightarrow 0 ; p_{j} \rightarrow 1$, and $p_{i}=p_{j}=$ 0.5 , we can assign it the intermediate evenness value of 0.5 . In the same manner, by averaging the $p_{i}=0.25 ; p_{j}=$
0.75 and the $p_{i}=p_{j}=0.5$ cases, we obtain the relative abundances $p_{i}=(0.25+0.5) / 2=0.375$ and $p_{j}=(0.75+$ $0.5) / 2=0.625$ to which we can assign the evenness value 0.75 . Iterating this procedure, we can assign evenness values to all possible cases of two-species communities. It is worth noticing that if the values obtained by this procedure are plotted against the relative abundance $p_{i}$, the outcome is a straight line (Figure 3). In this way, since we may know a priori the evenness values obtained from any community composed of two species, we can use twospecies communities to evaluate the performance of any evenness measure intended to be applied to species-rich communities. For instance, if we compare two multi-species communities using an evenness index with an ideal Molinari shape (i.e., with a linear response ranging from minimum evenness up to unity), it can be argued that, since the index keeps a linear relationship to evenness, the differences obtained in the resulting values are due to actual differences in community organization, rather than to index values bearing a non-linear relationship to evenness (Molinari 1989). Among the selected evenness measures, only the index of Bulla $O$ and the Gini index $I$ display a linear Molinari shape (Smith and Wilson 1996).

However, it is easily shown that in the most extreme case of a dominant species whose proportional abundance is very close to one, the minimum value assumed by both $O$ and $I$ approaches $1 / N$. Therefore, both indices lack the desirable property of varying between zero and one. In particular, for a two-species community, $O_{\min }=I_{\min }=0.5$. This shortcoming obviously cannot be solved by simple index normalization. For instance, the resulting normalized evenness index $E_{n}=(E-1 / N) /(1-1 / N)$ is not invariant under species replication violating the foremost requirement for an ecologically meaningful evenness index.

\section{Conclusion}

We tested here the mutual relatedness of seven standard measures of evenness. Principal component analysis 
was used to identify two individual components that represent the most important aspects of the distribution of abundance among community species. We further suggest that these two principal components can be represented in a simpler way by two evenness measures. The second principal component is clearly associated to changes in the abundance of the dominant species and can be best represented by $E_{\infty, 0}$. Conversely, regarding the first principal component, the choice depends to some extent on the user's requirements: if a linear Molinari shape is important, the Gini index is the most adequate choice due to its higher coefficient of variation with respect to the index of Bulla (see Table 1). If the Molinari shape is not important, due to its direct interpretation in information theoretical terms, our recommendation for surrogating the first principal component is $E_{1,0}$.

Acknowledgments: The authors wish to thank J. Podani and two anonymous referees for the stimulating comments that substantially contributed to improving this work.

\section{References}

Alatalo, R.V. 1981. Problems in the measurement of evenness in ecology. Oikos 37: 199-204.

Basak, S.C., A.T. Balaban, G.D. Grunwald and B.D. Gute 2000 Topological indices: their nature and mutual relatedness. $J$. Chem. Inf. Comput. Sci. 40: 891-898.

Bulla, L. 1994. An index of evenness and its associated diversity measure. Oikos 70: 167-171.

Camargo, J.A. 1992. Can dominance influence stability in competitive interactions? Oikos 64: 605-609.

He, X.S. and L. Orlóci 1993. Comparative diversity analysis of vegetation. Abstr. Bot. 17: 79-86.

Hill, M.O. 1973. Diversity and evenness: a unifying notation and its consequences. Ecology 54: 427-431.

Lande, R. 1996. Statistics and partitioning of species diversity, and similarity among multiple communities. Oikos 76: 5-13.
Magurran, A. 1988. Ecological Diversity and its Measurement. Princeton University Press, Princeton, NJ.

Molinari, J. 1989. A calibrated index for the measurement of evenness. Oikos 56: 319-326.

Patil, G.P. and C. Taillie 1979. An overview of diversity. In: J.F. Grassle, G.P. Patil, W.K. Smith and C. Taillie (eds.), Ecological Diversity in Theory and Practice. International Cooperative Publishing House, Fairland, MD, pp. 3-27.

Patil, G.P. and C. Taillie, C. 1982. Diversity as a concept and its measurement. J. Am. Stat. Assoc. 77: 548-567.

Peet, R.K. 1974. The measurement of species diversity. Ann. Rev. Ecol. Syst. 5: 285-307.

Podani, J. 1993. Multivariate Data Analysis in Ecology and Systematics. A Methodological Guide to SYN-TAX Package. Ecological Computations Series, Vol. 6, SPB Publishing, The Hague.

Rényi, A. 1970. Probability Theory. North Holland Publishing Company, Amsterdam.

Ricotta, C. and G.C. Avena 2000. A parametric index of community evenness. Ecoscience 7: 511-515.

Riitters, K.H., R.V. O’Neill, C.T. Hunsaker, J.D. Wickham, D.H. Yankee, S.P. Timmins, K.B. Jones and B.L. Jackson. 1995. A factor analysis of community pattern and structure metrics. Landscape Ecol. 10: 23-39.

Rousseau, R., P. vanHecke, D. Nijssen and J. Bogaert 1999. The relationship between diversity profiles, evenness and species richness based on partial ordering. Environ. Ecol. Stat. 6: 211-223.

Routledge, R.D. 1983. Evenness indices: are any admissible? Oikos 40: 149-151.

Sheldon, A.L. 1969. Equitability indices: dependence on the species count. Ecology 50: 466-467

Smith, B. and J.B. Wilson 1996. A consumer's guide to evenness indices. Oikos 76: 70-82.

Taillie, C. 1979. Species equitability: a comparative approach. In: J.F. Grassle, G.P. Patil, W.K. Smith and C. Taillie (eds.), Ecological Diversity in Theory and Practice. International Cooperative Publishing House, Fairland, MD, pp. 51-62. 